\title{
RETRACTED ARTICLE: Biologically validating the measurement of oxytocin in western lowland gorilla (Gorilla gorilla gorilla) urine and saliva using a commercial enzyme immunoassay
}

\author{
Austin Leeds ${ }^{1,2}$ (D) Patricia M. Dennis ${ }^{1,2,3} \cdot$ Kristen E. Lukas ${ }^{1,2} \cdot$ Tara S. Stoinski $^{4} \cdot$ Mark A. Willis $^{2} \cdot$ Mandi W. Schook $^{1,2,5}$
}

Received: 14 August 2017 / Accepted: 27 January 2018 / Published online: 8 February 2018

( ) Japan Monkey Centre and Springer Japan KK, part of Springer Nature 2018

The authors are retracting this article because an error in the reported methodology of this study was found when a follow up investigation of gorilla oxytocin was undertaken. The methods section of this article reports that an extraction step was used in preparing the urine and saliva samples for assay; however, a critical step in the extraction process was mistakenly omitted. The data reported here thus represent a validation of unextracted samples and not of extracted samples. Additional analysis is currently being conducted to understand how the unextracted samples compare to extracted samples and we will submit a new manuscript for review when this has been completed. All authors agree with this retraction.

The online version of this article contains the full text of the retracted article as electronic supplementary material.

Electronic supplementary material The online version of this article (https://doi.org/10.1007/s10329-018-0651-1) contains supplementary material, which is available to authorized users.

Austin Leeds

cal@clevelandmetroparks.com

1 Cleveland Metroparks Zoo, 3900 Wildlife Way, Cleveland, $\mathrm{OH} 44109$, USA

2 Department of Biology, Case Western Reserve University, Cleveland, OH, USA

3 Department of Veterinary Preventive Medicine, The Ohio State University, Columbus, OH, USA

4 Dian Fossey Gorilla Fund International, Atlanta, GA, USA

5 Disney's Animals, Science and Environment, Bay Lake, FL, USA 